\title{
Vitamin B12 deficiency presenting as an acute confusional state: a case report and review of literature
}

\author{
*Kibirige D, Wekesa C, Kaddu-Mukasa M, Waiswa M
}

Department of Medicine, Makerere University College of Health Sciences and Haematology Unit, Mulago National Referral and Teaching hospital, Kampala Uganda

\begin{abstract}
Background: Vitamin B12 deficiency is associated with a wide spectrum of neuro-psychiatric manifestations. Results: We report a case of a 44 year old female patient referred to the haematology unit with vitamin B12 deficiency presenting as an acute confusional state or delirium. Total resolution of the psychiatric symptoms occurred following parenteral vitamin B12 replacement therapy.

Conclusion: This case report highlights one of the neuro-psychiatric presentations of vitamin B12 deficiency in a previously healthy individual.

Key words: Vitamin B12 deficiency, acute confusional state, delirium.

African Health Sciences 2013; 13(3): 850 - 852 http://dx.doi.org/10.4314/ahs.v13i3.47
\end{abstract}

\section{Introduction}

Vitamin B12 deficiency has been highly linked to several psychiatric disorders like impaired memory, irritability, depression, dementia, delirium, schizophrenia and psychosis ${ }^{1}$. Other commonly associated neurological disorders include paraesthesias, numbness and sub acute combined degeneration of the spinal cord ${ }^{2}$.

Varying prevalence of vitamin B12 deficiency has been reported among patients with different psychiatric conditions. Published studies have documented prevalence of $29 \%, 44 \%$ and $70.8 \%$ among patients with primary dementia ${ }^{3}$, depression ${ }^{4}$ and schizophrenia ${ }^{5}$ respectively.

In Africa, Maktouf et al in a multi centre prospective study in Tunisia found a prevalence of vitamin B12 deficiency of $14 \%$ among 82 psychiatric patients ${ }^{6}$. A recent study done in Uganda demonstrated prevalence of low vitamin B12 levels of 28.6\% among 280 psychiatric patients admitted at the main psychiatric referral hospital 7 . One percent of these patients had delirium as the primary psychiatric diagnosis.

*Corresponding author:
Dr. Davis Kibirige
Department of Medicine
College of Health Sciences, Makerere University
P.O. Box 7062 Kampala, Uganda
Tel: 256414541188
Mobile: +256782 393955
E-mail: davouirek@yahoo.co.uk.

\section{Case report}

A 44 year old HIV sero negative lady was referred to the haematology unit from a rural hospital mainly for blood transfusion due to severe anemia. She presented with a three weeks history of uncoordinated speech, aggressiveness, reduced sleep, wandering away from her home, poor concentration and visual hallucinations mainly worsening at night. The above symptoms were preceded by a three months history of generalised body weakness, intense paraesthesias of the lower limbs, palpitations and exertional dyspnea.

She did not have any past psychiatric history, history suggestive of diabetes mellitus, auto immune diseases or any past gastric or ileal surgery. She was a peasant farmer residing about 600 kilometres from the hospital and was not a vegetarian. Her daily diet was predominantly carbohydrate rich with minimal intake of animal protein.

Physical examination revealed severe pallor of the mucous membranes and atrophic tongue papillae. She was fully awake but disoriented in time and place, looked sad with poor concentration, incoherent speech and short term memory impairment. Neurological examination revealed diminished tendon reflexes globally and impaired position and vibration sense of the first metatarsophalangeal joint bilaterally. Haematological tests included a complete blood count (CBC) which showed severe anemia of $5 \mathrm{~g} / \mathrm{dl}$ (normal: 12-16) with a raised mean cell volume of 119 (normal: 80-100), 
thrombocytopenia of 137,000 (normal: 150,000 $400,000)$ and normal white blood cell count. The peripheral film examination showed macrocytes with polychromasia. Serum vitamin B12 levels were very low at $62.94 \mathrm{pg} / \mathrm{ml}$ (normal: 204-946). Cerebrospinal fluid examination, liver and renal function tests were all normal. Red blood cell folate levels and antibodies to the intrinsic factor were not done due to financial constraints.

A diagnosis of delirium or acute confusional state secondary to vitamin B12 deficiency probably due to inadequate dietary intake was made. The patient was started on intramuscular vitamin B12 1 mg daily for one week which was followed by 1 mg monthly with oral iron and folate therapy for a week. She was also transfused with two units of whole blood. There was complete resolution of the symptoms and improvement in the $\mathrm{CBC}$ indices within one week of administration of the vitamin B12 while on the ward. The patient was fully counselled about her condition and discharged later.

\section{Discussion}

This case report illustrates an acute confusional state or delirium as a manifestation of vitamin B12 deficiency. Such psychiatric conditions can be the solitary or preliminary manifestations of vitamin B12 deficiency and may precede haematological signs by several months or years ${ }^{8}$.

A serum vitamin B12 level of less than $200 \mathrm{pg} / \mathrm{ml}$ is diagnostic of vitamin B12 deficiency ${ }^{9}$ as shown in the patient discussed. However, measuring serum levels of methylmalonic acid (MMA) or total homocysteine have also been found to be more sensitive biochemical indicators of vitamin B12 deficiency especially among people with trivial or no haematological abnormalities ${ }^{10}$.

Several mechanisms have been described to explain neuro cognitive dysfunction due to vitamin B12 deficiency. Vitamin B12 is essential in the synthesis of monoamines like serotonin and dopamine ${ }^{11}$. It is also very integral in the methylation process of homocysteine to methionine which is activated into S-adenosyl-methionine that donates its methyl group to methyl acceptors such as myelin, neurotransmitters and membrane phospholipids ${ }^{12}$. Metabolically significant vitamin B12 deficiency results in disruption of the methylation process and intracellular accumulation of homocysteine that is potentially toxic to neurones ${ }^{11-12}$.

The most common cause of vitamin B12 deficiency is insufficient dietary intake especially among alcoholics and vegetarians. Other causes include atrophic gastritis, mainly in the elderly, pernicious anemia, malabsorption syndromes; and drug induced (e.g. metformin and proton pump inhibitors (PPIs) $)^{2,8}$.

Clinical evaluation of patients with vitamin B12 deficiency should include a thorough drug history, assessment for malabsorption syndromes, and screening for autoimmune causes of vitamin B12 deficiency, such as pernicious anemia. Much of this information can be gathered based on clinical history and physical exam ${ }^{8}$. In resource limited settings, obtaining ancillary testing (e.g. anti-parietal cell and anti-intrinsic factor antibodies) may be a challenge due to high test costs or lack of availability of tests.

The presumed cause of vitamin B12 deficiency in this patient was inadequate dietary intake of Vitamin B12 rich foods which is very common among people living in the rural areas of Uganda. She also did not have any history of chronic PPI or metformin use or any clinical evidence suggestive of auto immune conditions and malabsorption syndrome.

Both parenteral and oral vitamin B12 replacement therapies have been demonstrated to have equivalent effectiveness in achieving the desirable haematological and neurological remissions regardless of the aetiology of vitamin B12 deficiency ${ }^{13-15}$. Intra muscular vitamin B12 treatment was preferred in this patient because it is cheaper in Uganda and also to improve on compliance.

\section{Conclusion}

This case report describes a previously healthy lady without past personal psychiatric history presenting with delirium secondary to vitamin B12 deficiency. It illustrates the importance of considering the possibility of vitamin B12 deficiency among patients with delirium and other psychiatric symptoms. Serum vitamin B12 determination in these patients should be encouraged as part of standard clinical evaluation.

\section{References}

1. Moore E, Mander A, Ames D, Carne R, Sanders K, Watters D. Cognitive impairment and vitamin B12: a review. Int Psychogeriatr 2012;116.

2. Robert C, David L. Vitamin B12 Deficiency. American Family Physician 2003;67:979-86.

3. Karnaze D, Carmel R. Low serum cobalamin levels in primary degenerative dementia: do 
some patients harbor atypical cobalamin deficiency states? Arch Intern Med 1987;147:42931.

4. Penninx BW, Guralnik JM, Ferrucci L, Fried LP, Allen RH, Stabler SP. Vitamin B12 deficiency and depression in physically disabled older women: epidemiologic evidence from the Women's Health and Aging Study. Am J Psychiatry 2000;157:715-21.

5. Silver H. Vitamin B12 levels are low in hospitalised psychiatric patients. Isr J Psychiatry Relat Sci 2000;37:41-5.

6. Maktouf C, Bchir F, Louzir H, et al. Clinical spectrum of cobalamin deficiency in Tunisia. Ann Biol Clin (Paris) 2007;65:135-42.

7. Michael S, Seggane M, D Herny Prevalence and factors associated with low Vitamin B12 levels among psychiatric patients admitted in Butabika mental hospital. Joint 7th Annual Scientific conference of Makerere University College of Health Sciences and the 19th Uganda National Association of Community and Occupational Health Abstract No: PS1016_21.

8. Andrès E, Loukili NH, Noel E. Vitamin B12 (cobalamin) deficiency in elderly patients. CMAJ 2004;171:251-9.

9. Snow C. Laboratory Diagnosis of vitamin B12 and folate deficiency. A guide for the primary care physician. Arch Intern Med 1999;159:128998.

10. Klee G. Cobalamin and folate evaluation: measurements of methylmalonic acid and homocystein vs vitamin B12 and folate. Clin Chem 2000;46:1277-83.

11. Bottiglieri T LM, Crellin R. Homocysteine, folate, methylation, and monoamine metabolism in depression. I Neurol Neurosurg Psychiatry 2000;69:228-32.

12. Malouf R AS. Vitamin B12 for cognition. Cochrane Database of Systematic Reviews 2003.

13. Vidal-Alaball J, Butler C, Cannings-John R, etal. Oral vitamin B12 versus parenteral vitamin B12 for vitamin B12 deficiency. The Cochrane Database of Systematic Reviews 2005.

14. Bolaman Z, Kadikoylu G, Yukselen V, et-al. Oral Versus Intramuscular Cobalamin Treatment in Megaloblastic Anemia: A SingleCenter, Prospective, Randomized, Open-Label Study. Clinical Therapentics 2003;25:3124-34.

15. Kuzminski A, Del-Giacco E, Allen R, Stabler S, Lindenbaum J. Effective Treatment of Cobalamin Deficiency With Oral Cobalamin. Blood 1998; 92:1191-8. 